\title{
Impact of frontline treatment approach on outcomes of myeloid blast phase CML
}

\author{
Kapil Saxena' ${ }^{1}$ Elias Jabbour², Ghayas Issa², Koji Sasaki², Farhad Ravandi², Abhishek Maiti², Naval Daver², \\ Tapan Kadia ${ }^{2}$, Courtney D. DiNardo², Marina Konopleva², Jorge E. Cortes ${ }^{3}$, Musa Yilmaz², Kelly Chien², \\ Sherry Pierce ${ }^{2}$, Hagop Kantarjian ${ }^{2}$ and Nicholas J. Short ${ }^{2^{*}}$ (D)
}

\begin{abstract}
Background: The natural course of untreated chronic myeloid leukemia $(\mathrm{CML})$ is progression to an aggressive blast phase. Even in the current era of BCR-ABL1 tyrosine kinase inhibitors (TKIs), the outcomes of blast phase CML remain poor with no consensus frontline treatment approach.

Methods: We retrospectively analyzed the response rates and survival outcomes of 104 consecutive patients with myeloid blast phase CML (CML-MBP) treated from 2000 to 2019 based on 4 different frontline treatment approaches: intensive chemotherapy (IC) +TKI $(n=20)$, hypomethylating agent (HMA) $+\operatorname{TKI}(n=20)$, TKI alone $(n=56)$, or IC alone $(n=8)$. We also evaluated the impact of TKI selection and subsequent allogeneic stem cell transplant (ASCT) on patient outcomes.
\end{abstract}

Results: Response rates were similar between patients treated with IC+TKI and HMA+TKI. Compared to treatment with TKI alone, treatment with IC/HMA + TKI resulted in a higher rate of complete remission (CR) or CR with incomplete count recovery (CRi) $(57.5 \%$ vs $33.9 \%, p<0.05)$, a higher complete cytogenetic response rate ( $45 \%$ vs $10.7 \%, p<0.001$ ), and more patients proceeding to ASCT (32.5\% vs $10.7 \%, p<0.01$ ). With a median follow-up of 6.7 years, long-term outcomes were similar between the IC + TKI and HMA + TKI groups. Combination therapy with IC/HMA + TKI was superior to therapy with TKI alone, including when analysis was limited to those treated with a 2nd/3rd-generation TKI. When using a 2nd/3rd-generation TKI, IC/HMA + TKI led to lower 5-year cumulative incidence of relapse $(\mathrm{CIR} ; 44 \%$ vs $86 \%, p<0.05)$ and superior 5 -year event-free survival (EFS; $28 \%$ vs $0 \%, p<0.05)$ and overall survival (OS; $34 \%$ vs $8 \%, p=0.23$ ) compared to TKI alone. Among patients who received IC/HMA + TKI, EFS and OS was superior for patients who received a 2 nd/3rd generation TKI compared to those who received imatinib-based therapy. In a landmark analysis, 5-year OS was higher for patients who proceeded to ASCT (58\% vs 22\%, $p=0.12$ ).

Conclusions: Compared to patients treated with TKI alone for CML-MBP, treatment with IC + TKI or HMA+TKI led to improved response rates, CIR, EFS, and OS, particularly for patients who received a 2nd/3rd-generation TKI. Combination therapy with IC + TKI or HMA + TKI, rather than a TKI alone, should be considered the optimal treatment strategy for patients with CML-MBP.

Keywords: CML, Blast phase, Chemotherapy, Hypomethylating agent, TKI

*Correspondence: nshort@mdanderson.org

2 Department of Leukemia, The University of Texas MD Anderson Cancer Center, 1515 Holcombe Blvd, Unit 0428, Houston, TX 77030, USA

Full list of author information is available at the end of the article

\section{Introduction}

Chronic myeloid leukemia (CML), which is characterized by the presence of the Philadelphia chromosome $(\mathrm{Ph}+)$ and the resultant $B C R-A B L 1$ fusion, occurs in different phases dependent on the absence or presence of certain original author(s) and the source, provide a link to the Creative Commons licence, and indicate if changes were made. The images or other third party material in this article are included in the article's Creative Commons licence, unless indicated otherwise in a credit line to the material. If material is not included in the article's Creative Commons licence and your intended use is not permitted by statutory regulation or exceeds the permitted use, you will need to obtain permission directly from the copyright holder. To view a copy of this licence, visit http://creativecommons.org/licenses/by/4.0/. The Creative Commons Public Domain Dedication waiver (http://creativeco mmons.org/publicdomain/zero/1.0/) applies to the data made available in this article, unless otherwise stated in a credit line to the data. 
clinical features, including cytogenetic clonal evolution, basophilia, and elevated blast percentage [1]. CML presents in a chronic phase (CP) in about $95 \%$ of patients [2]. Approximately $5 \%$ of patients present with an advanced phase of disease, either in an accelerated (AP) or blast phase (BP) [2]. The natural history of untreated or TKIresistant CML is progression to $\mathrm{BP}$ (with or without an intervening AP) within 3-8 years from diagnosis of CML-CP, with a rapidly fatal disease course upon onset of $\mathrm{BP}[3-5]$.

BCR-ABL1 TKIs have remarkably altered the prognosis of CML, with patients in CP now having a normal lifespan compared to their age-matched peers and the prospect of a cure within reach for those who enter a durable treatment-free remission (TFR) [6, 7]. In the pivotal phase III IRIS study comparing imatinib to chemoimmunotherapy (cytarabine + IFNo), the 10-year rate of progression to an advanced phase was reduced from $12.8 \%$ with chemoimmunotherapy to $6.9 \%$ with imatinib [8]. The 10-year cumulative incidence of BP in the large imatinib-based CML-study IV cohort was $5.8 \%$, and this rate has reduced even further with the use of secondgeneration TKIs (dasatinib, nilotinib, bosutinib) in the frontline setting [9-12].

Unlike CML-CP, patients who either present with de novo CML-BP or progress to $\mathrm{BP}$ from a previous $\mathrm{CP} / \mathrm{AP}$ still have dismal outcomes, and the median OS remains less than 1 year after diagnosis of BP [13]. Approximately $70-80 \%$ of BP cases occur in myeloid blast phase (MBP) and $20-30 \%$ occur as a lymphoid blast phase (LBP) or with blasts of mixed lineage $[1,5]$. Patients with LBP have better outcomes than those with MBP [14]. For CML-MBP, treatment approaches vary from the use of single-agent TKI to acute myeloid leukemia (AML)like induction therapy + TKI, and there is no consensus frontline treatment recommendation [13, 15-17]. In order to clarify the optimal therapy for these patients, we retrospectively analyzed all patients receiving frontline treatment for CML-MBP over the past 20 years at our institution and assessed response rates, depth of response, and survival outcomes across different frontline therapies.

\section{Methods}

\section{Study design and participants}

This was a retrospective study including patients $\geq 18$ years of age treated in the frontline setting for CML-MBP between 2000 and 2019. MBP was defined as the presence of $\geq 20 \%$ bone marrow (BM) myeloblasts with or without concurrent extramedullary (EM) disease per the World Health Organization 2017 criteria for CML-BP. Patients could have received prior therapy (including TKIs) for CML in $\mathrm{CP}$ /
AP. Exclusion criteria were the presence of EM-only disease or any prior therapy for CML-BP aside from hydroxyurea. Patients were divided into four frontline treatment approaches: (1) intensive chemotherapy (IC) + TKI, (2) hypomethylating agent (HMA) + TKI, (3) TKI alone, or (4) IC alone. IC was defined as a regimen containing $\geq 1 \mathrm{~g} / \mathrm{m}^{2}$ of cytarabine. All patients in the HMA + TKI group received decitabine. For certain specified analyses, groups 1 and 2 were combined into an "IC/HMA + TKI" group. TKI therapy was divided into 1st-generation (imatinib), 2nd-generation (dasatinib, nilotinib, bosutinib), or 3rd-generation (ponatinib) agents. The retrospective study was conducted at a single academic medical center (The University of Texas MD Anderson Cancer Center), approved by the Institutional Review Board, and conducted in accordance with the Declaration of Helsinki.

\section{Response and outcome definitions}

Morphologic/hematologic responses were assessed per AML response criteria, as defined according to European LeukemiaNet consensus guidelines [18]. Patients who achieved a best response of complete remission (CR), complete remission with incomplete hematological recovery (CRi), or morphologic leukemia-free state (MLFS) were considered responders, and all others were considered nonresponders. Complete cytogenetic response (CCyR) was defined as no detectable $\mathrm{Ph}+$ chromosome by conventional karyotyping. Major molecular response (MMR; $\mathrm{MR}^{3}$ ) was defined as a $B C R-A B L 1 / A B L 1$ ratio $>0.01$ and $\leq 0.1 \%$ on the International Scale (IS). $\mathrm{MR}^{4}$ was defined as a $B C R-A B L 1 /$ $A B L 1$ ratio $>0.0032 \%$ and $\leq 0.01 \%$ on the International Scale (IS). $\mathrm{MR}^{4.5}$ was defined as a $B C R-A B L 1 /$ $A B L 1$ ratio $\leq 0.0032 \%$ on the International Scale (IS). All molecular responses were assessed by quantitative real-time PCR analysis at the molecular diagnostics laboratory at MD Anderson Cancer Center and were converted to the IS.

Relapse was defined as $\geq 5 \%$ BM blasts, new extramedullary disease, or appearance of peripheral blasts unrelated to BM recovery after an initial objective response. Cumulative incidence of relapse (CIR) was calculated from time of initial response (CR, CRi, MLFS) to relapse, censored for death in morphologic remission or if the patient was alive at last follow-up. Event-free survival (EFS) was defined as the time from diagnosis to lack of response, morphologic relapse after initial morphologic response, or death from any cause. Overall survival (OS) was defined as the time from diagnosis to death from any cause. EFS and OS were censored if the patient was alive at last follow-up. 


\section{Statistical methods}

Patient characteristics were analyzed using descriptive statistics, and survival analyses were performed using Kaplan-Meier methodology. To compare two groups, Chi-Square test was performed for categorical variables, and $t$-test or Mann-Whitney test was performed for continuous variables. Log-rank (Mantel-Cox) test was performed to compare Kaplan-Meier survival curves. All statistical analyses were conducted using GraphPad Prism version 8.4.3.

\section{Results}

\section{Patient characteristics and study cohort}

Between January 2000 and April 2019, 104 patients received frontline treatment for CML-MBP at our institution and met our inclusion criteria. Twenty patients were treated with IC + TKI, 20 with HMA + TKI, 56 with TKI alone, and 8 with IC alone. Baseline patient characteristics of each group are shown in Table 1 . Patients in the IC + TKI group were generally younger than patients in the 3 other groups (median age: 47 years vs. 57 years, respectively). Overall, 91 patients (87.5\%) progressed from a prior CP/AP, and 67 (64.4\%) had received at least one prior TKI for preceding $\mathrm{CP} /$ AP. Prior exposure to chemotherapy and/or HMA for CML CP/AP is detailed in Additional file 1: Table 1. Nine patients $(8.6 \%)$ had concurrent EM disease in addition to BM involvement at the time of MBP diagnosis. The most common additional chromosomal abnormalities (ACAs) were +8 , extra $\mathrm{Ph}$, and $3 \mathrm{q} 26$ rearrangements; T315I was the most common $A B L 1$ mutation (Additional file 1: Tables 2, 3).

In the IC+TKI cohort, all patients received a 2nd/3rd-generation TKI as part of their initial CMLMBP therapy. In contrast, $65 \%$ and $52 \%$ of patients in

Table 1 Baseline patient characteristics at time of CML-MBP treatment initiation

\begin{tabular}{|c|c|c|c|c|}
\hline \multirow[t]{2}{*}{ Characteristic } & \multicolumn{4}{|c|}{$N(\%) ;$ median [range] } \\
\hline & $\begin{array}{l}\text { IC }+ \text { TKI } \\
(N=20)\end{array}$ & $\mathrm{HMA}+\mathrm{TKI}(N=20)$ & $\begin{array}{l}\text { TKI } \\
(N=56)\end{array}$ & $\begin{array}{l}\mathrm{IC} \\
(N=8)\end{array}$ \\
\hline Age, years & $47[29-83]$ & $56[37-89]$ & $57[21-79]$ & $56[27-74]$ \\
\hline \multicolumn{5}{|l|}{ Race/ethnicity } \\
\hline White, non-Hispanic & $10(50 \%)$ & $14(70 \%)$ & $37(66 \%)$ & $5(62.5 \%)$ \\
\hline White, Hispanic & $4(20 \%)$ & $1(5 \%)$ & $2(3.6 \%)$ & 0 \\
\hline Black & $4(20 \%)$ & $4(20 \%)$ & $14(25 \%)$ & $3(37.5 \%)$ \\
\hline Other & 0 & 0 & $1(1.8 \%)$ & 0 \\
\hline Not stated & $2(10 \%)$ & $1(5 \%)$ & $2(3.6 \%)$ & 0 \\
\hline Initial CML presentation as de novo MBP & $5(25 \%)$ & $5(25 \%)$ & $3(5.3 \%)$ & 0 \\
\hline Year of treatment initiation for MBP & $\begin{array}{l}2013 \\
{[2007-2018]}\end{array}$ & $\begin{array}{l}2013 \\
{[2003-2019]}\end{array}$ & $\begin{array}{l}2004 \\
{[2000-2012]}\end{array}$ & $\begin{array}{l}2003 \\
{[2000-2003]}\end{array}$ \\
\hline Prior regimens for $\mathrm{CML}^{*}$ & $1[0-3]$ & $1[0-4]$ & $1.5[0-5]$ & $3[0-4]$ \\
\hline Prior TKI exposure & $14(70 \%)$ & $14(70 \%)$ & $32(60 \%)$ & $7(87.5 \%)$ \\
\hline Changed TKI for MBP & $10 / 14$ & $7 / 14$ & $30 / 32$ & N/A \\
\hline BM blasts (\%) & $39[21-87]$ & $52[24-91]$ & $47[20-87]$ & $30(20-60)$ \\
\hline EM disease at diagnosis^ & $3 / 20(15 \%)$ & $0 / 20(0 \%)$ & $4 / 56(7.1 \%)$ & $2 / 8(25 \%)$ \\
\hline Additional clonal cytogenetic abnormalities & $12(60 \%)$ & $15(75 \%)$ & $42(75 \%)$ & $6(75 \%)$ \\
\hline T315I mutation & $3 / 20(15 \%)$ & $0 / 14(0 \%)$ & $1 / 23(4.3 \%)$ & N/A \\
\hline $\mathrm{WBC}\left(\times 10^{9} / \mathrm{L}\right)$ & $21.9[3.1-259.3]$ & $37.7[1.0-156.6]$ & $23.8[0.7-363.7]$ & $32.4[2.4-319]$ \\
\hline Platelet $\left(\times 10^{9} / \mathrm{L}\right)$ & $127[7-607]$ & $75[12-431]$ & $82[7-1128]$ & $52[21-2750]$ \\
\hline \multicolumn{5}{|l|}{ Initial TKI for MBP } \\
\hline Imatinib & 0 & $7(35 \%)$ & $26(48 \%)$ & $\mathrm{N} / \mathrm{A}$ \\
\hline Dasatinib & $10(50 \%)$ & $11(55 \%)$ & $12(21 \%)$ & $\mathrm{N} / \mathrm{A}$ \\
\hline Nilotinib & $2(10 \%)$ & $1(5 \%)$ & $12(21 \%)$ & N/A \\
\hline Bosutinib & $1(5 \%)$ & $0(0 \%)$ & $3(5 \%)$ & N/A \\
\hline Ponatinib & $7(35 \%)$ & $1(5 \%)$ & $3(5 \%)$ & N/A \\
\hline
\end{tabular}

IC intensive chemotherapy, HMA hypomethylating agent, WBC white blood cell count, TKI tyrosine kinase inhibitor, MBP myeloid blast phase, $B M$ bone marrow, EM extramedullary disease

*Not including hydroxyurea

$\wedge$ biopsy-confirmed 
the HMA + TKI and TKI alone groups, respectively, received a $2 \mathrm{nd} / 3 \mathrm{rd}$-generation TKI as their initial TKI for CML-MBP. Treatment regimens and year of therapy initiation are shown in Additional file 1: Table 4. The median year of MBP treatment initiation was 2013 for both IC + TKI and HMA + TKI compared to 2003 and 2004 for TKI alone or IC, respectively. These differences in therapies reflect a change in institutional practice around 2009 to preferentially use combination therapy with IC/HMA + TKI, rather than TKI alone, for patients with CML-MBP.

Table 2 Outcomes

\begin{tabular}{|c|c|c|c|c|}
\hline \multirow[t]{2}{*}{ Characteristic } & \multicolumn{4}{|c|}{ N (\%); Median [Range] } \\
\hline & $\begin{array}{l}\text { IC+TKI } \\
(N=20)\end{array}$ & HMA + TKI $(N=20)$ & $\begin{array}{l}\text { TKI } \\
(N=56)\end{array}$ & $\begin{array}{l}\mathrm{IC} \\
(N=8)\end{array}$ \\
\hline \multicolumn{5}{|l|}{ Best response } \\
\hline$C R$ & $9(45 \%)$ & $6(30 \%)$ & $12(21.4 \%)$ & 0 \\
\hline CRi & $3(15 \%)$ & $5(25 \%)$ & $7(12.5 \%)$ & $1(12.5 \%)$ \\
\hline MLFS & $4(20 \%)$ & $3(15 \%)$ & $8(14.3 \%)$ & 0 \\
\hline$P R$ & 0 & 0 & $1(1.8 \%)$ & 0 \\
\hline No response & $4(20 \%)$ & $6(30 \%)$ & $28(50 \%)$ & $7(87.5 \%)$ \\
\hline $\mathrm{CR} / \mathrm{CRi}$ & $12(60 \%)$ & $11(55 \%)$ & $19(33.9 \%)$ & $1(12.5 \%)$ \\
\hline ORR (CR/CRi/MLFS) & $16(80 \%)$ & $14(70 \%)$ & $27(48.2 \%)$ & $1(12.5 \%)$ \\
\hline Complete cytogenetic remission & $8(40 \%)$ & $10(50 \%)$ & $6(10.7 \%)$ & 0 \\
\hline \multicolumn{5}{|l|}{ Best molecular response $\wedge$} \\
\hline MMR & $2 / 17(11.7 \%)$ & $1 / 16(6.3 \%)$ & $1 / 47(2.1 \%)$ & 0 \\
\hline $\mathrm{MR}^{4}$ & $2 / 17(11.7 \%)$ & $2 / 16(12.5 \%)$ & 0 & 0 \\
\hline $\mathrm{MR}^{4.5}$ & $1 / 17(5.9 \%)$ & 0 & $1 / 47(2.1 \%)$ & 0 \\
\hline Time to best response (months) & $\begin{array}{l}0.9 \\
{[0.7-6.9]}\end{array}$ & $\begin{array}{l}2.2 \\
{[0.8-5.5]}\end{array}$ & $\begin{array}{l}2.1 \\
{[0.6-16.3]}\end{array}$ & $\begin{array}{l}0.6 \\
{[0.6-0.6]}\end{array}$ \\
\hline Proceeded to ASCT on this regimen & $7(35 \%)$ & $6(30 \%)$ & $6^{*}(10.7 \%)$ & $1(12.5 \%)$ \\
\hline Median time to ASCT (months) & $3.4[1.5-7.9]$ & $5.7[2.9-8]$ & $3.5[2.5-5.7]$ & $1.3[1.3-1.3]$ \\
\hline Median EFS (months) & $\begin{array}{l}5.2 \\
{[0.8-160.7]}\end{array}$ & $\begin{array}{l}5.0 \\
{[1.2-96.1]}\end{array}$ & $\begin{array}{l}4.8 \\
{[0.5-129.6]}\end{array}$ & $\begin{array}{l}2.2 \\
{[0.8-4.1]}\end{array}$ \\
\hline Median RFS (months) & $\begin{array}{l}5.5 \\
{[0.5-159.8]}\end{array}$ & $\begin{array}{l}4.7 \\
{[0.6-93.5]}\end{array}$ & $\begin{array}{l}4.6 \\
{[0.2-127.7]}\end{array}$ & $\begin{array}{l}3.5 \\
{[3.5-3.5]}\end{array}$ \\
\hline Median OS (months) & $\begin{array}{l}12.9 \\
{[0.8-160.7]}\end{array}$ & $\begin{array}{l}10.1 \\
{[1.2-96.1]}\end{array}$ & $\begin{array}{l}10.7 \\
{[0.5-244.3]}\end{array}$ & $\begin{array}{l}3.4 \\
{[0.8-48.9]}\end{array}$ \\
\hline Relapse after initial objective response & $6 / 16$ & $6 / 14$ & $17 / 27$ & $0 / 1$ \\
\hline EM relapse ${ }^{* *}$ & $3 / 6(50 \%)$ & $1 / 6(16.7 \%)$ & $3 / 17(17.6 \%)$ & $0 / 1(0 \%)$ \\
\hline \multicolumn{5}{|l|}{ Early mortality } \\
\hline 30-day mortality & $1(5 \%)$ & 0 & $1(1.8 \%)$ & $1(12.5 \%)$ \\
\hline 60-day mortality & $3(15 \%)$ & $2(10 \%)$ & $1(1.8 \%)$ & $3(37.5 \%)$ \\
\hline \multicolumn{5}{|l|}{ 5-year rates } \\
\hline $\mathrm{CIR}$ & $51 \%$ & $54 \%$ & $80 \%$ & $100 \%$ \\
\hline EFS & $27 \%$ & $19 \%$ & $5 \%$ & $0 \%$ \\
\hline OS & $30 \%$ & $28 \%$ & $13 \%$ & $0 \%$ \\
\hline
\end{tabular}

$\mathrm{CR}$, complete remission; $\mathrm{CRi}$, complete remission with incomplete count recovery; $\mathrm{PR}$, partial remission; MLFS, morphologic leukemia-free state; ORR, overall response rate; ASCT, allogeneic stem cell transplantation; $\mathrm{MMR}$, major molecular response; $\mathrm{CMR}$, complete molecular response; CIR, cumulative incidence of relapse; $\mathrm{EFS}$, eventfree survival; RFS, relapse-free survival; OS, overall survival

$\wedge$ censored at time of SCT or first event, definitions below:

MMR $\left(\mathrm{MR}^{3}\right): B C R-A B L 1>0.01 \%$ to $\leq 0.1 \%$ on the international scale (IS)

$M R^{4}: B C R-A B L 1>0.0032 \%$ to $\leq 0.01 \%$ on the IS

$M R^{4.5}: B C R-A B L 1 \leq 0.0032 \%$ on the IS

*2 of the 6 patients in the TKI group went to ASCT with active disease

**Including central nervous system (CNS) relapse; one patient had CNS relapse in IC/TKI cohort, all other EM relapses were outside the CNS 


\section{Response rates}

Morphologic, cytogenetic, and molecular responses rates are shown in Table 2. Patients treated with IC alone had the lowest response rate with only one objective response $(\mathrm{CRi})$ among $8 \mathrm{IC}$-treated patients. Overall, responses rates were similar in patients treated with IC + TKI or HMA + TKI (CR/CRi rates $60 \%$ vs $55 \%$, CCyR rates $40 \%$ vs $50 \%$, MMR or deeper molecular response rates $29.4 \%$ vs $18.8 \%$, respectively). Given similar responses between the IC + TKI and HMA + TKI groups, we combined these two groups for additional analyses $(n=40)$. Compared to treatment with TKI alone, combination therapy (IC/HMA + TKI) resulted in higher rates of $\mathrm{CR} / \mathrm{CRi}(57.5 \%$ vs $33.9 \%, p<0.05)$, CCyR (45\% vs $10.7 \%, p<0.001)$, and MMR or deeper molecular response $(24.2 \%$ vs $4.3 \%, p<0.01)$.

\section{Relapse and survival outcomes}

Relapse and survival outcomes are shown in Table 2. Overall, the 5-year CIR rate was similar in patients who received $\mathrm{IC}+\mathrm{TKI}$ or $\mathrm{HMA}+\mathrm{TKI}$, and the 5-year CIR rate was lower in patients treated with either IC + TKI or HMA + TKI compared to patients treated with TKI alone (Fig. 1a). The lower CIR with IC/HMA + TKI was observed in both the entire cohort and in the subgroup of patients treated with a 2nd/3rd-generation TKI (Fig. 1b). For patients treated with combination therapy (IC/HMA + TKI), the 5-year CIR rate was significantly lower compared to TKI monotherapy when a $2 \mathrm{nd} / 3 \mathrm{rd}$ generation TKI was used $(44 \%$ vs $86 \%, p=0.02)$ (Fig. 1c).

Though median EFS and OS were similar between the IC + TKI, HMA + TKI, and TKI alone groups (Table 2), long-term survival outcomes differed among the treatment groups. With median follow-up of 6.7 years, 5-year EFS and OS was superior for patients treated with combination therapy compared to those treated with TKI alone (Fig. 2a, b). The superior outcomes with IC/ HMA + TKI were observed despite a higher 60-day mortality for patients treated with combination therapy compared to TKI alone ( $12.5 \%$ vs $1.8 \%, p<0.05$, Table 2 ; cause of 60-day mortality listed in Additional file 1: Table 5). When limited to patients treated with a 2 nd/3rd-generation TKI, 5-year EFS was 0\% for those treated with TKI alone, 27\% with IC + TKI, and 29\% with HMA + TKI (Fig. 2c). 5-year OS was 8\% for those treated with TKI alone, $30 \%$ with IC + TKI, and 38\% with HMA+TKI, (Fig. 2d). Among patients who received a 2 nd/3rd-generation TKI, combination therapy with IC/HMA + TKI resulted in improved 5-year EFS (28\% vs $0 \%, p<0.05$; Fig. 2e) and 5-year OS (34\% vs $8 \%, p=0.23$; Fig. 2f) compared to treatment with TKI alone.
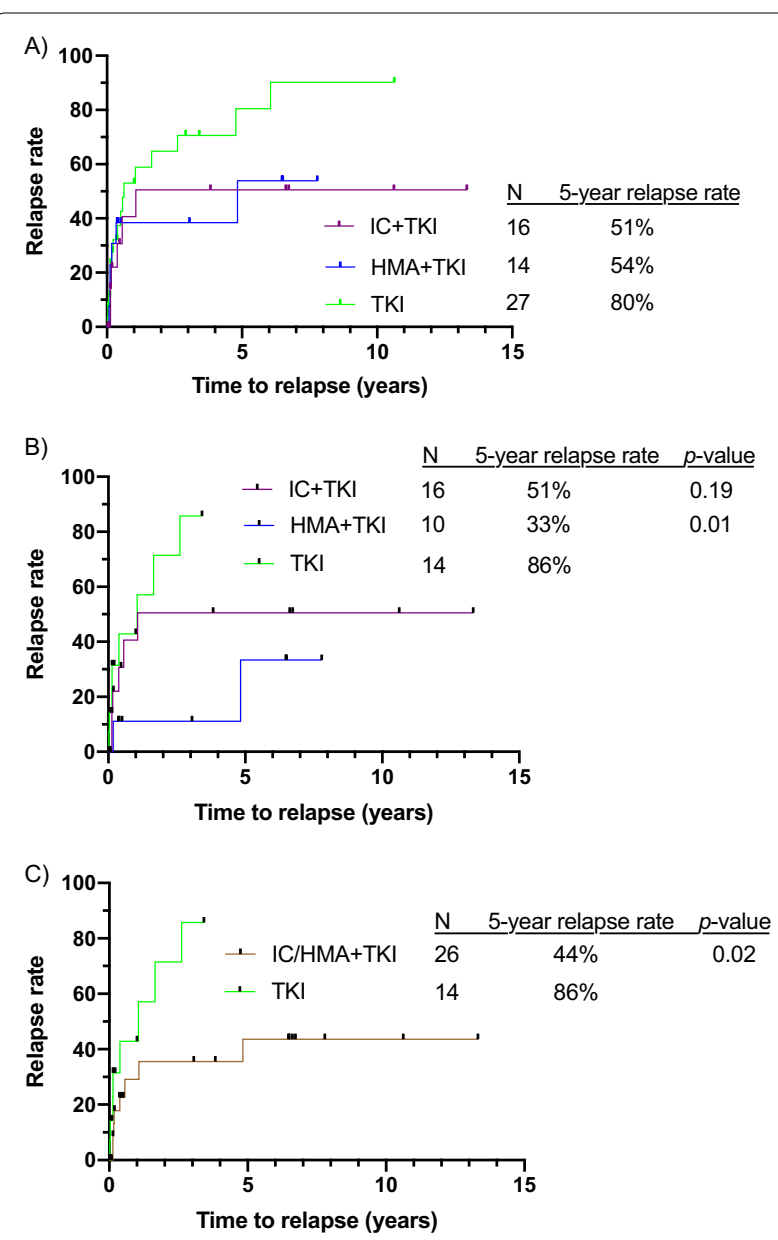

Fig. 1 Cumulative incidence of relapse (CIR) based on a therapeutic approach including (a) any TKI or ( $\mathbf{b}$ and $\mathbf{c}$ ) only including regimens with a 2 nd/3rd-generation TKI. $p$-values are between the indicated group and the TKI monotherapy group

Lastly, the effect of ACAs on clinical outcome was examined. Given that all 4 groups had a similar number of patients with at least one ACA (60\% in IC+TKI, $75 \%$ in the other 3 groups), patients with at least $1 \mathrm{ACA}$ $(n=75)$ were compared to patients without an ACA $(n=29)$. As has been described previously, the presence of ACAs conferred worse EFS and OS compared to no ACAs (Additional file 1: Fig. 1) [19].

Impact of TKI received on response and survival outcomes Given the increasing use of a 2nd/3rd-generation TKI in the treatment of CML-MBP as opposed to an imatinibbased regimen, we assessed outcomes for patients treated with IC/HMA + TKI based on TKI generation. Of the 40 patients treated with IC/HMA + TKI, 7 were treated with an imatinib-based regimen, 25 with a 2 ndgeneration TKI-based regimen, and 8 with a ponatinibbased regimen. Four out of 57 tested patients (7\%) had 

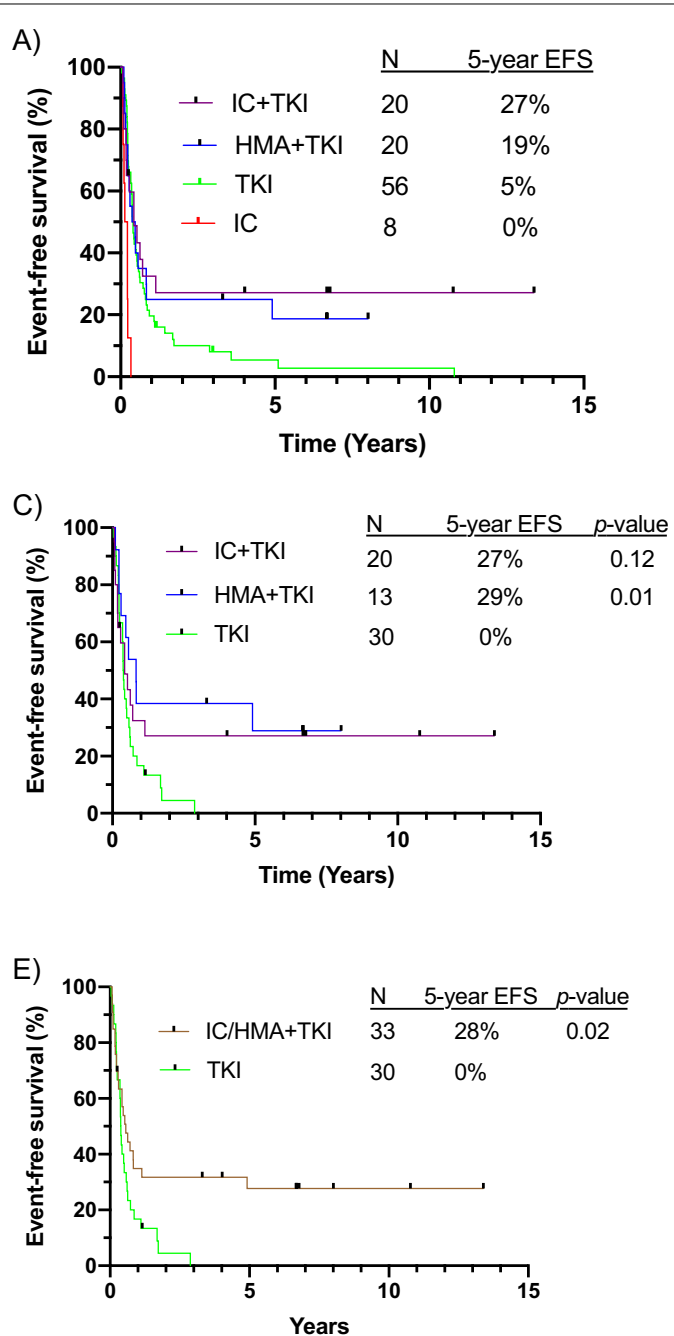
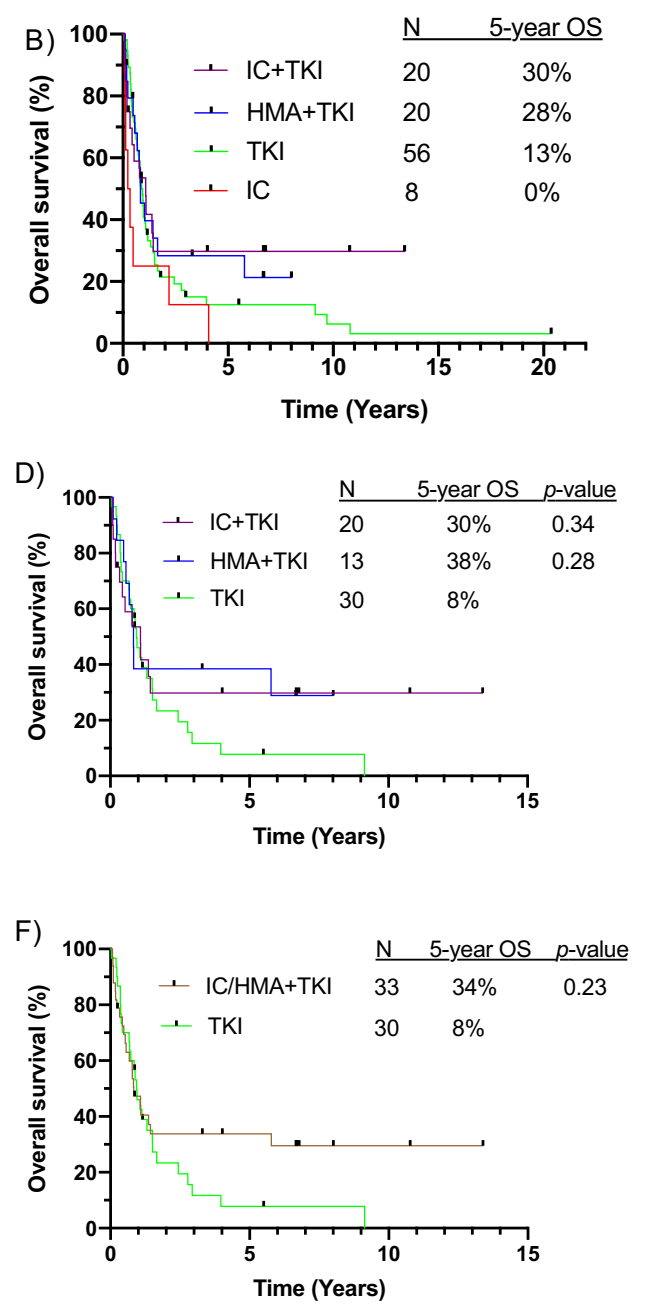

Fig. 2 Outcomes based on any therapeutic approach for (a) event-free survival (EFS) and (b) overall survival (OS). Outcomes for patients who received 2nd/3rd-generation TKI for (c) EFS and (d) OS. Outcomes for patients who received combination therapy versus TKI monotherapy for (e) EFS and (f) OS). $p$-values are between the indicated group and the TKI monotherapy group

Table 3 Outcomes with IC/HMA + TKI based on TKI generation

\begin{tabular}{|c|c|c|c|}
\hline \multirow[t]{2}{*}{ Outcome } & \multicolumn{3}{|l|}{$N(\%)$} \\
\hline & $\begin{array}{l}\text { IC/HMA }+ \\
\text { imatinib } \\
(N=7)\end{array}$ & $\begin{array}{l}\text { IC/HMA+ } \\
\text { 2nd generation } \\
\text { TKI }(N=25)\end{array}$ & $\begin{array}{l}\text { IC/HMA+ } \\
\text { ponatinib } \\
(N=8)\end{array}$ \\
\hline$C R$ & $1(14.3 \%)$ & $11(44 \%)$ & $3(37.5 \%)$ \\
\hline $\mathrm{CR} / \mathrm{CRi}$ & $2(28.6 \%)$ & $16(64 \%)$ & $5(62.5 \%)$ \\
\hline CR/CRi/MLFS & $4(57.1 \%)$ & $19(76 \%)$ & $7(87.5 \%)$ \\
\hline 5-year CIR & $100 \%$ & $45 \%$ & $38 \%$ \\
\hline 5-year EFS & $0 \%$ & $25 \%$ & $38 \%$ \\
\hline 5-year OS & $0 \%$ & $32 \%$ & $38 \%$ \\
\hline
\end{tabular}

$\mathrm{CR}$, complete remission; $\mathrm{CRi}$, complete remission with incomplete count recovery; MLFS, morphologic leukemia-free state;

$\mathrm{CIR}$, cumulative incidence of relapse; $\mathrm{EFS}$, event-free survival; OS, overall survival a T315I mutation at the time of CML-MBP diagnosis (Table 1 ), and $1 / 8$ patients treated with ponatinib had a detectable T315I mutation. Response rates and survival outcomes by TKI are shown in Table 3. Imatinib-based combination therapy was associated with very poor outcomes, with 5-year EFS and OS rates of $0 \%$ compared to 5 -year EFS and OS rates of $25 \%$ and $32 \%$, respectively, with a 2nd-generation TKI-containing regimen, and 5 -year EFS and OS rates of $38 \%$ and 38\%, respectively, with a ponatinib-based regimen (Fig. 3a, b).

\section{Impact of ASCT on survival outcomes}

Treatment with combination therapy (IC/HMA + TKI) led to more patients proceeding to ASCT compared to TKI alone $(32.5 \%$ vs $10.7 \%, p<0.01)$. Median time 

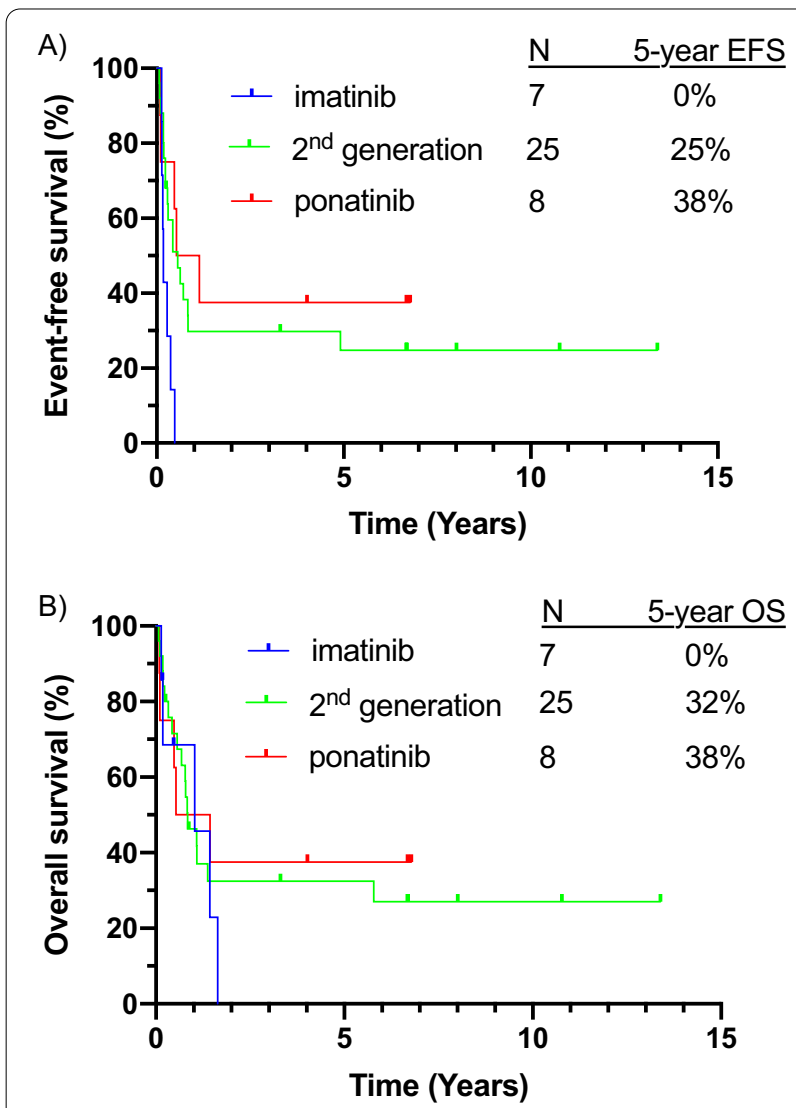

Fig. 3 Outcomes stratified by TKI received for patients treated with combination therapy plus a TKI for (a) event-free survival and (b) overall survival

to ASCT was 4.7 months in the entire cohort of patients and 4.9 months for patients treated with IC/ HMA + TKI. Landmark survival analysis was performed on patients with a morphologic response (i.e. CR, CRi, or MLFS) who were alive and event-free at the landmark time point. Those who underwent ASCT $(n=19)$ had superior OS compared to those who did not $(n=22)$, with 5 -year OS rates of $58 \%$ vs. $22 \%(p=0.12)$ (Fig. 4a). Similar benefit to ASCT was seen when only including patients treated with $\mathrm{IC} /$ HMA + TKI (Fig. 4b).

Notably, 4 patients survived $>5$ years despite not undergoing ASCT. One patient was treated with IC + TKI followed by maintenance TKI, 1 patient with HMA + TKI followed by maintenance TKI, and 2 patients with imatinib alone. Of the 4 patients, only one relapsed. This patient was treated with imatinib initially followed by different single-agent TKI
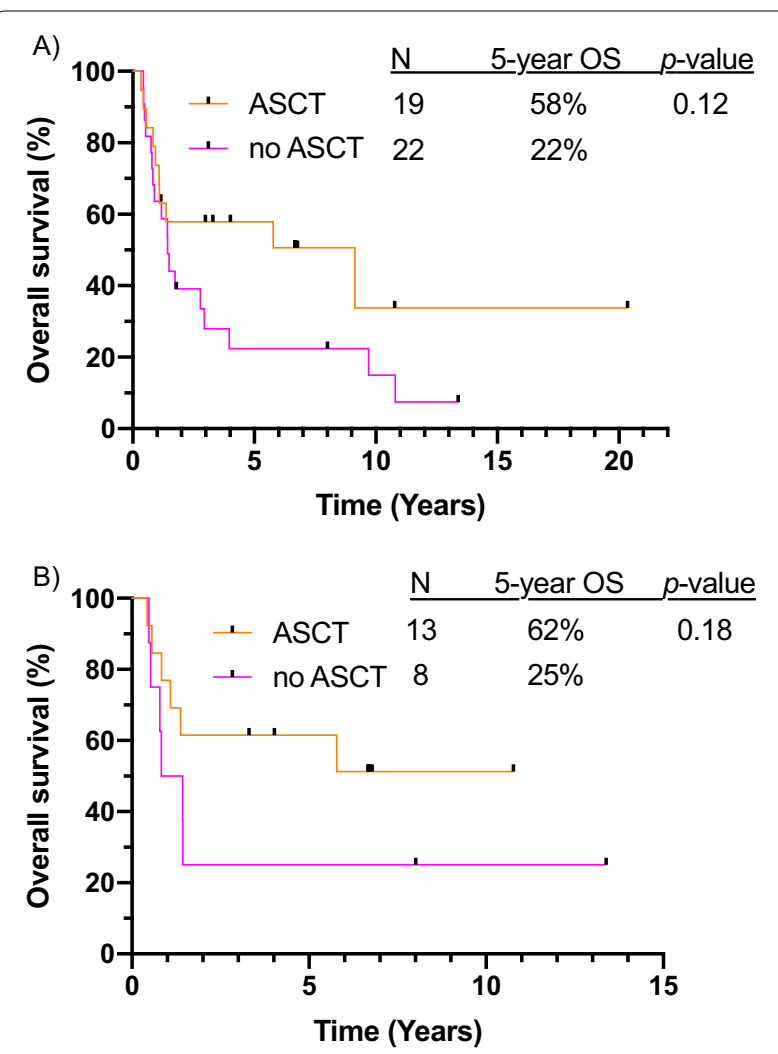

Fig. 4 Landmark overall survival analysis for transplanted versus non-transplanted patients, including (a) all patients in the cohort and (b) only those who received combination therapy plus a TKI

regimens due to treatment intolerance and ultimately relapsed 6 years after initial MBP diagnosis.

\section{Discussion}

Outcomes for CML-CP have improved considerably in the TKI era [13]. However, the occurrence of BP remains a challenging clinical scenario and for many patients is an end-stage event [6]. Due to its rarity, treatment is guided primarily by retrospective series, small singlearm clinical trials, and expert opinion. In this study, we identified 104 patients with CML-MBP over a 20 -year period treated with different therapeutic approaches in the frontline setting. To our knowledge, this is the largest cohort of patients with CML-MBP to include data on response rates, relapse incidence, and survival based on therapeutic approach. We found that therapy with IC/ HMA + TKI in comparison to TKI alone yields higher response rates, lower risk of relapse, and improved 5-year EFS/OS, suggesting that combination therapy with IC or HMA plus a TKI should be consider the optimal upfront therapy for these patients, rather than TKI alone.

Although there are several published algorithms on how to approach CML-MBP, there is no formal category 
1 recommendation in clinical guidelines. Trial enrollment, AML-like induction therapy + TKI, or TKI alone are all considered acceptable treatment options [17]. The incorporation of a TKI is crucial, as the BCR-ABL1 fusion kinase remains a significant leukemic driver in patients with CML-BP. For historical comparison to demonstrate this point, we included patients in our study treated with IC alone. While the number of patients in our cohort who received IC alone is small, it is evident that IC alone is inadequate therapy, with a response rate of $12.5 \%$ and 5-year EFS and OS rates of $0 \%$. The utilization of TKI monotherapy instead of IC improved outcomes for patients, however long-term outcomes remained poor with a 5 -year EFS rate of $5 \%$ and OS rate of $13 \%$. While the subsequent use of combination regimens (IC/ HMA + TKI) did not significantly improve median EFS/ OS compared to TKI, there was a clear benefit in the proportion of patients achieving long-term survival.

The utilization of combination therapy for CML-MBP may have yielded improved responses compared to TKI alone because progression to MBP, with or without the development of a resistant $A B L 1$ mutation, suggests development of leukemogenic events independent of the BCR-ABL1 kinase $[5,20]$. The biology of BP is much less understood compared to CP. Patients with BP may have mutations in genes commonly associated with AML, such as ASXL1 and RUNX1, as well as ACAs that confer a negative prognosis in AML, such as -7 and inv(3) $[19,21,22]$. These mutations and ACAs along with ongoing constitutive BCR-ABL1 kinase activity and additional cellular changes yield a complex disease biology in which both proliferative and anti-apoptotic pathways have been implicated, including but not limited to those mediated by Wnt/ $\beta$-catenin, MYC, and BCL-2/BCL-xL $[5,23]$. The progression to MBP therefore appears to create a more heterogenous disease than CML-CP, and targeting a single driver pathway may be insufficient. Given the proliferation of myeloblasts and immature progenitor cells, aspects of the disease clinically appear to mimic AML more so than CML-CP. Thus, the use of an acute leukemia chemotherapeutic regimen + TKI may be efficacious by affecting multiple cell survival/death mechanisms rather than one primary target, as has been shown for patients with $\mathrm{Ph}+$ acute lymphoblastic leukemia (ALL) treated with TKI-based combination regimens [24].

Patients treated with combination therapy had improved long-term survival in our cohort, and it appears that the etiology of this benefit primarily arose from a higher rate of patients proceeding to ASCT (32.5\% with IC/HMA + TKI vs. $10.7 \%$ with TKI alone). Among patients who had a clinical response and underwent ASCT, 5 -year OS was $58 \%$ for all patients $(62 \%$ for those treated with IC/HMA+TKI). Prior to the TKI era, ASCT was the only curative option for patients with any phase of CML, and it remains the therapeutic goal for patients with CML-BP [25]. In AML, Ph + ALL, and CML-CP, outcomes post-ASCT are influenced by the pre-transplant depth of response [26-28]. TKI therapy alone resulted in a CR/CRi rate of $33.9 \%$ and CCyR rate of $10.7 \%$. In contrast, combination therapy produced a $\mathrm{CR} / \mathrm{CRi}$ in $57.5 \%$ of patients and CCyR in $45 \%$ of patients. MMR or deeper molecular response rates were relatively low with combination therapy $(29.4 \%$ with IC + TKI and $18.8 \%$ with HMA + TKI) but were still higher than the 4.3\% rate with TKI alone. Thus, it is possible that the improved outcomes associated with IC/HMA+TKI were due to a higher likelihood of reducing leukemia burden pre-transplant, thereby permitting more patients to proceed to the potentially curative option of ASCT.

IC + TKI and HMA + TKI have been assessed separately in small, early phase clinical trials and retrospective studies [29-34]. However, to the best of our knowledge they have not been previously compared to each other within the same cohort. Though IC + TKI and HMA + TKI produced similar response rates and survival outcomes in this cohort, it is notable that patients in the IC+TKI group were younger, only received a 2nd/3rd-generation TKI, and were more likely to receive ponatinib compared to patients treated with HMA + TKI. Though 60-day mortality was similar between both groups, HMAs are typically better tolerated with fewer toxicities compared to most IC regimens for AML. With regards to which TKI to use in combination therapy, no patient treated with an imatinib-based combination regimen survived past 2 years, further supporting the use of later-generation TKIs in this context. Assessing for differences between patients treated with ponatinib or a 2ndgeneration TKI is limited by the small number of patients treated with ponatinib $(n=8)$. However, 5-year EFS and OS rates were similar or slightly higher with the use of ponatinib compared to 2nd-generation TKIs, which parallels findings in $\mathrm{Ph}+\mathrm{ALL}$ where the use of ponatinib as the frontline TKI has demonstrated significant clinical efficacy even in the absence of a T315I mutation $[35,36]$. Ongoing studies are evaluating lower-intensity therapy (HMA + venetoclax) combined with ponatinib for patients with $\mathrm{Ph}+$ myeloid malignancies, including CML-MBP (NCT04188405), which may further improve outcomes for this disease. Retrospective and prospective studies also support the use of venetoclax-based combination regimens in CML-BP and advanced $\mathrm{Ph}+$ leukemias [37, 38].

Our study is limited primarily due to its retrospective, nonrandomized, single-center methodology. Thus, we were unable to capture certain types of clinical information that are typically gathered for prospective 
clinical studies and may have influenced treatment outcomes, such as patient performance status, physician rationale for treatment choice, and adverse events. Due to the declining incidence of CML-MBP, prospective randomized trials are unlikely to occur and, if so, will likely require significant time to accrue. We aimed to minimize the potential biases inherent in a retrospective cohort analysis by having relatively strict inclusion criteria and focusing on patients receiving their first therapy for CML-MBP and excluding patients with EM-only disease. We focused on patients who received frontline treatment for CML-MBP in order to minimize the variability associated with including patients within the same analysis who had no prior BP treatment with those who had relapsed/refractory disease to one or more prior BP regimens. For patients with EM-only disease, responses as assessed by imaging rather than typical CML or AML hematologic and molecular criteria would have complicated our response assessment, particularly in a retrospective analysis where imaging modalities were not consistently used.

\section{Conclusion}

In summary, our study supports the use of combination therapy with IC + TKI or HMA + TKI rather than TKI monotherapy for patients with CML-MBP. IC + TKI and HMA + TKI produced similar results, with long-term survival of approximately $30 \%$, and superior outcomes in those patients who were able to proceed to ASCT. In particular, the combination of HMA + 2nd/3rd-generation TKI is highly effective, despite being less intensive than an IC-based approach. These data provide a valuable benchmark for future prospective studies evaluating novel therapies for patients with CML-MBP.

\section{Supplementary Information}

The online version contains supplementary material available at https://doi. org/10.1186/s13045-021-01106-1.

Additional file 1. Supplementary Tables 1-5 and Supplementary Figure 1.

\section{Acknowledgements}

Not applicable.

\section{Authors' contributions}

K.S and N.J.S. designed the study, collected and analyzed the data, performed the statistical analyses, and wrote the manuscript. S.P collected the data. All other authors treated patients. All authors reviewed and approved the manuscript.

\section{Funding}

Supported by an MD Anderson Cancer Center Support Grant (CA016672) and SPORE. N.J.S. is supported by the K12 Paul Calabresi Clinical Oncology Scholar
Award and the American Society of Hematology Junior Faculty Scholar Award in Clinical Research.

\section{Availability of data and materials}

All data generated and analyzed during this study are included in this published article and the supplementary files.

\section{Declarations}

\section{Ethics approval and consent to participate}

The retrospective study was conducted at a single academic medical center (The University of Texas MD Anderson Cancer Center), approved by the Institutional Review Board, and conducted in accordance with the Declaration of Helsinki.

\section{Consent for publication}

Not applicable.

\section{Competing interests}

N.J.S has served as a consultant for Takeda Oncology, AstraZeneca and Jazz Pharmaceuticals, reports receiving research grants from Takeda Oncology and Astellas Pharma Inc., and has received honoraria from Amgen. The other authors report no relevant conflicts of interest.

\section{Author details}

${ }^{1}$ Division of Cancer Medicine, MD Anderson Cancer Center, Houston, TX, USA. ${ }^{2}$ Department of Leukemia, The University of Texas MD Anderson Cancer Center, 1515 Holcombe Blvd, Unit 0428, Houston, TX 77030, USA. ${ }^{3}$ Georgia Cancer Center, Augusta, GA, USA.

Received: 19 April 2021 Accepted: 7 June 2021

Published online: 15 June 2021

\section{References}

1. Swerdlow SH, Campo E, Harris NL, Jaffe ES, Pileri S, Stein H, et al. WHO classification of tumours of haematopoietic and lymphoid tissues. Lyon: International Agency for Research on Cancer; 2017.

2. Hoffmann VS, Baccarani M, Hasford J, Lindoerfer D, Burgstaller S, Sertic $D$, et al. The EUTOS population-based registry: incidence and clinical characteristics of 2904 CML patients in 20 European Countries. Leukemia. 2015;29(6):1336-43.

3. Sawyers CL. Chronic myeloid leukemia. N Engl J Med. 1999;340(17):1330-40.

4. Hehlmann R. CML-Where do we stand in 2015? Ann Hematol. 2015;94(Suppl 2):S103-5.

5. Chereda B, Melo JV. Natural course and biology of CML. Ann Hematol. 2015;94(Suppl 2):S107-21.

6. Hehlmann R. Chronic Myeloid Leukemia in 2020. Hemasphere. 2020;4(5):468.

7. Saussele S, Richter J, Guilhot J, Gruber FX, Hjorth-Hansen H, Almeida A, et al. Discontinuation of tyrosine kinase inhibitor therapy in chronic myeloid leukaemia (EURO-SKI): a prespecified interim analysis of a prospective, multicentre, non-randomised, trial. Lancet Oncol. 2018;19(6):747-57.

8. Hochhaus A, Larson RA, Guilhot F, Radich JP, Branford S, Hughes TP, et al. Long-term outcomes of imatinib treatment for chronic myeloid leukemia. N Engl J Med. 2017;376(10):917-27.

9. Hehlmann R, Lauseker M, Saussele S, Pfirrmann M, Krause S, Kolb HJ, et al. Assessment of imatinib as first-line treatment of chronic myeloid leukemia: 10-year survival results of the randomized CML study IV and impact of non-CML determinants. Leukemia. 2017;31(11):2398-406.

10. Kantarjian HM, Hughes TP, Larson RA, Kim DW, Issaragrisil S, le Coutre $\mathrm{P}$, et al. Long-term outcomes with frontline nilotinib versus imatinib in newly diagnosed chronic myeloid leukemia in chronic phase: ENESTnd 10-year analysis. Leukemia. 2021;35(2):440-53.

11. Cortes JE, Saglio G, Kantarjian HM, Baccarani M, Mayer J, Boque C, et al. Final 5 -year study results of dasision: the dasatinib versus imatinib study in treatment-naive chronic myeloid leukemia patients trial. J Clin Oncol. 2016;34(20):2333-40. 
12. Brummendorf TH, Cortes JE, Milojkovic D, Gambacorti-Passerini C, Clark $R E$, le Coutre PD, et al. Bosutinib (BOS) versus imatinib for newly diagnosed chronic phase (CP) chronic myeloid leukemia (CML): final 5-year results from the bfore trial. Blood. 2020;136:41-2.

13. Hehlmann R, Saussele S, Voskanyan A, Silver RT. Management of CMLblast crisis. Best Pract Res Clin Haematol. 2016;29(3):295-307.

14. Jain P, Kantarjian HM, Ghorab A, Sasaki K, Jabbour EJ, Nogueras Gonzalez $G$, et al. Prognostic factors and survival outcomes in patients with chronic myeloid leukemia in blast phase in the tyrosine kinase inhibitor era: cohort study of 477 patients. Cancer. 2017;123(22):4391-402.

15. Bonifacio M, Stagno F, Scaffidi L, Krampera M, Di Raimondo F. Management of chronic myeloid leukemia in advanced phase. Front Oncol. 2019:9:1132.

16. Hochhaus A, Baccarani M, Silver RT, Schiffer C, Apperley JF, Cervantes F, et al. European LeukemiaNet 2020 recommendations for treating chronic myeloid leukemia. Leukemia. 2020;34(4):966-84.

17. NCCN. Chronic Myeloid Leukemia (Version 3.2021) 2021. https://www. nccn.org/professionals/physician_gls/pdf/cml.pdf.

18. Dohner H, Estey E, Grimwade D, Amadori S, Appelbaum FR, Buchner T, et al. Diagnosis and management of AML in adults: 2017 ELN recommendations from an international expert panel. Blood. 2017;129(4):424-47.

19. Wang W, Cortes JE, Tang G, Khoury JD, Wang S, Bueso-Ramos CE, et al. Risk stratification of chromosomal abnormalities in chronic myelogenous leukemia in the era of tyrosine kinase inhibitor therapy. Blood. 2016;127(22):2742-50.

20. Hamilton A, Helgason GV, Schemionek M, Zhang B, Myssina S, Allan EK, et al. Chronic myeloid leukemia stem cells are not dependent on Bcr-Abl kinase activity for their survival. Blood. 2012;119(6):1501-10.

21. Ochi Y, Yoshida K, Huang YJ, Kuo MC, Nannya Y, Sasaki K, et al. Clonal evolution and clinical implications of genetic abnormalities in blastic transformation of chronic myeloid leukaemia. Nat Commun. 2021:12(1):2833.

22. Branford S, Wang P, Yeung DT, Thomson D, Purins A, Wadham C, et al. Integrative genomic analysis reveals cancer-associated mutations at diagnosis of CML in patients with high-risk disease. Blood. 2018;132(9):948-61.

23. Perrotti D, Jamieson C, Goldman J, Skorski T. Chronic myeloid leukemia: mechanisms of blastic transformation. J Clin Invest. 2010;120(7):2254-64.

24. Samra B, Jabbour E, Ravandi F, Kantarjian H, Short NJ. Evolving therapy of adult acute lymphoblastic leukemia: state-of-the-art treatment and future directions. J Hematol Oncol. 2020;13(1):70.

25. Barrett AJ, Ito $\mathrm{S}$. The role of stem cell transplantation for chronic myelogenous leukemia in the 21st century. Blood. 2015;125(21):3230-5.

26. Oran B, Jorgensen JL, Marin D, Wang S, Ahmed S, Alousi AM, et al. Pretransplantation minimal residual disease with cytogenetic and molecular diagnostic features improves risk stratification in acute myeloid leukemia. Haematologica. 2017;102(1):110-7.

27. Lussana F, Intermesoli T, Gianni F, Boschini C, Masciulli A, Spinelli O, et al. Achieving molecular remission before allogeneic stem cell transplantation in adult patients with philadelphia chromosome-positive acute lymphoblastic leukemia: impact on relapse and long-term outcome. Biol Blood Marrow Transplant. 2016;22(11):1983-7.
28. Oehler VG, Gooley T, Snyder DS, Johnston L, Lin A, Cummings CC, et al. The effects of imatinib mesylate treatment before allogeneic transplantation for chronic myeloid leukemia. Blood. 2007;109(4):1782-9.

29. Abaza Y, Kantarjian H, Alwash Y, Borthakur G, Champlin R, Kadia T, et al. Phase I/II study of dasatinib in combination with decitabine in patients with accelerated or blast phase chronic myeloid leukemia. Am J Hematol. 2020;95(11):1288-95.

30. Copland M, Slade D, Byrne J, Brock K, De Lavallade H, Craddock C, et al. FLAG-IDA and ponatinib in patients with blast phase chronic myeloid leukaemia: results from the phase I/II UK trials acceleration programme matchpoint trial. Blood. 2019;134:497.

31. Deau B, Nicolini FE, Guilhot J, Huguet F, Guerci A, Legros L, et al. The addition of daunorubicin to imatinib mesylate in combination with cytarabine improves the response rate and the survival of patients with myeloid blast crisis chronic myelogenous leukemia (AFR01 study). Leuk Res. 2011;35(6):777-82.

32. Milojkovic D, Ibrahim A, Reid A, Foroni L, Apperley J, Marin D. Efficacy of combining dasatinib and FLAG-IDA for patients with chronic myeloid leukemia in blastic transformation. Haematologica. 2012;97(3):473-4.

33. Oki Y, Kantarjian HM, Gharibyan V, Jones D, O'Brien S, Verstovsek S, et al. Phase II study of low-dose decitabine in combination with imatinib mesylate in patients with accelerated or myeloid blastic phase of chronic myelogenous leukemia. Cancer. 2007;109(5):899-906.

34. Ruggiu M, Oberkampf F, Ghez D, Cony-Makhoul P, Beckeriche F, Cano I, et al. Azacytidine in combination with tyrosine kinase inhibitors induced durable responses in patients with advanced phase chronic myelogenous leukemia. Leuk Lymphoma. 2018;59(7):1659-65.

35. Jabbour E, DerSarkissian M, Duh MS, McCormick N, Cheng WY, McGarry $\mathrm{L}$, et al. Efficacy of ponatinib versus earlier generation tyrosine kinase inhibitors for front-line treatment of newly diagnosed philadelphiapositive acute lymphoblastic leukemia. Clin Lymphoma Myeloma Leuk. 2018;18(4):257-65.

36. Jabbour E, Short NJ, Ravandi F, Huang X, Daver N, DiNardo CD, et al. Combination of hyper-CVAD with ponatinib as first-line therapy for patients with Philadelphia chromosome-positive acute lymphoblastic leukaemia: long-term follow-up of a single-centre, phase 2 study. Lancet Haematol. 2018;5(12):e618-27.

37. Maiti A, Franquiz MJ, Ravandi F, Cortes JE, Jabbour EJ, Sasaki K, et al. Venetoclax and BCR-ABL tyrosine kinase inhibitor combinations: outcome in patients with philadelphia chromosome-positive advanced myeloid leukemias. Acta Haematol. 2020;143(6):567-73.

38. Short NJ, Konopleva M, Kadia T, Kebriaei P, Daver N, Huang X, et al. An effective chemotherapy-free regimen of ponatinib plus venetoclax for relapsed/refractory Philadelphia chromosome-positive acute lymphoblastic leukemia. Am J Hematol. 2021.

\section{Publisher's Note}

Springer Nature remains neutral with regard to jurisdictional claims in published maps and institutional affiliations.

\footnotetext{
Ready to submit your research? Choose BMC and benefit from:

- fast, convenient online submission

- thorough peer review by experienced researchers in your field

- rapid publication on acceptance

- support for research data, including large and complex data types

- gold Open Access which fosters wider collaboration and increased citations

- maximum visibility for your research: over 100M website views per year
}

At $\mathrm{BMC}$, research is always in progress.

Learn more biomedcentral.com/submissions 